\title{
A CLASS OF BILINEAR FORMS
}

\author{
BY \\ HOWARD A. OSBORN
}

1. Introduction. Let $K$ be an arbitrary field of characteristic $\neq 2, E$ a finite dimensional vector space over $K$, and $A$ an endomorphism of $E$. What is the structure of the class $\mathrm{e}(A)$ of bilinear forms $f$ on $E \times E$ such that $A$ is self-adjoint with respect to $f$ ? The forms in $\mathfrak{C}(A)$ need not be positive definite in any sense, or even symmetric; the only requirement is that $f(A u, v)$ $=f(u, A v)$ for all $(u, v) \in E \times E$.

It will be convenient to treat two related problems first. Define the bilinear form $f A$ by setting $f A(u, v)=f(u, A v)$, let $s(A)$ denote the class of symmetric forms $f$ such that $f A$ is also symmetric, and let $Q(A)$ denote the class of alternating forms $f$ such that $f A$ is also alternating. Then $\delta(A)$ and $Q(A)$ may be described explicitly, and one easily shows that $\mathfrak{e}(A)=\mathfrak{S}(A) \oplus_{K} \mathbb{Q}(A)$, which solves the original problem.

Let $\mathbb{E}(A)$ denote the centralizer of $A$ in the ring $\Re$ of all endomorphisms of $E$. Then $\mathfrak{C}(A)$ is a right $\mathbb{E}(A)$-module in a natural way, which is isomorphic to the right $\mathbb{S}(A)$-module $\mathbb{S}(A)$. This isomorphism may be developed further by considering $\mathfrak{C}(A)$ and $\mathfrak{S}(A)$ as modules for the ring $\mathfrak{A}$ of endomorphisms of $E$ which is generated by $A$ and scalar multiplications. By means of the induced $\mathfrak{A}$-isomorphism one easily describes $\mathfrak{S}(A)$ as an $\mathfrak{A}$-module, obtaining Frobenius' theorem on $\operatorname{dim}_{K} \mathfrak{E}(A)$ as a trivial corollary. In fact, $\mathfrak{S}(A)$ may be considered as an algebra over $\mathfrak{A}$, that is, $\mathfrak{S}(A)$ admits $\mathfrak{A}$ as a ring of operators, and the submodules $\mathfrak{S}(A)$ and $\mathfrak{\&}(A)$ which are $\mathfrak{A}$-isomorphic to $\mathfrak{S}(A)$ and $\mathfrak{Q}(A)$, respectively, become Jordan and Lie algebras over $\mathfrak{A}$, with the usual definitions of products. Multiplication tables may be written out explicitly.

The final portions of the paper are devoted to matrix representations of $\mathfrak{e}(A)$ and $\mathfrak{E}(A)$.

The author wishes to thank Professor Nathan Jacobson for his interest in the problem.

2. The vector spaces $\mathfrak{S}(A), \mathfrak{a}(A)$, and $\mathfrak{e}(A)$. Let $\mathfrak{A}$ be the commutative ring of endomorphisms of $E$ which is generated by $A$ and scalar multiplications, and regard $E$ as an $\mathfrak{A}$-module. $E$ is cyclic if it contains an element $z$ such that $\mathfrak{A} z=E$, and a classical decomposition theorem states that in any event $E$ is the direct sum of cyclic submodules, $E=E_{1} \oplus \cdots \oplus E_{t}$, such that if $\Im\left(E_{i}\right)$ is the order ideal of $E_{i}$, then $i \leqq j$ implies that $\Im\left(E_{i}\right) \supseteq \Im\left(E_{j}\right)$. The decomposition is unique except in the case of submodules of equal dimension over $K$.

Presented to the Society, June 15, 1957; received by the editors May 10, 1957. 
Since each $E_{i}$ is cyclic, there exists an element $z_{i} \in E_{i}$, called an $E_{i}$ generator, such that $\mathfrak{A} z_{i}=\mathfrak{A} / \mathfrak{\Im}\left(E_{i}\right) z_{i}=E_{i}, i=1, \cdots, t$. We shall assume that $z_{1}, \cdots, z_{t}$ have been chosen once and for all.

LEMma 1. There is a unique isomorphism $u_{i} \rightarrow u_{i}^{*}$ of $E_{i}$ onto $\mathfrak{A} / \Im\left(E_{i}\right)$ such that $u_{i}^{*} z_{i}=u_{i}$ for any $u_{i} \in E_{i}$.

Proof. Clearly any such map is a homomorphism of $E_{i}$ into $\mathfrak{A} / \Im\left(E_{i}\right)$, and at least one $u_{i}^{*}$ exists, since $z_{i}$ is an $E_{i}$-generator. Suppose that $B z_{i}=0$ for some $B \in \mathfrak{A}$. Then $B E_{i}=B \mathfrak{Y} z_{i}=\mathfrak{A} B z_{i}=0$ so that $B \in \mathfrak{S}\left(E_{i}\right)$. Finally, to prove that the isomorphism is onto, let $B$ be any element of $\mathfrak{A} / \Im\left(E_{i}\right)$, and set $B z_{i}=y_{i}$. Then for any $x_{i} \in E_{i}, B x_{i}=B x_{i}^{*} z_{i}=x_{i}^{*} B z_{i}=x_{i}^{*} y_{i}=x_{i}^{*} y_{i}^{*} z_{i}=y_{i}^{*} x_{i}^{*} z_{i}$ $=y_{i}^{*} x_{i}$, so that $B=y_{i}^{*}$.

Clearly $z_{i}^{*}$ is the identity of $\mathfrak{A} / \Im\left(E_{i}\right)$ and $u_{i}^{*} v_{i}=u_{i}^{*} v_{i}^{*} z_{i}=v_{i}^{*} u_{i}^{*} z_{i}=v_{i}^{*} u_{i}$. Furthermore, if $i \leqq j, u_{j}^{*} v_{i}$ is a well-defined element of $E_{i}$, since $\Im\left(E_{i}\right) \supseteq \Im\left(E_{j}\right)$.

Lemma 2. If $B \in \mathfrak{A} / \Im\left(E_{i}\right)$ and $u_{i} \in E_{i}$, then $\left(B u_{i}\right)^{*}=B u_{i}^{*}$.

Proof. Let $v_{i}$ be any element of $E_{i}$. Then $\left(B u_{i}\right) v_{i}=\left(B u_{i}\right){ }^{*} v_{i}^{*} z_{i}=v_{i}^{*}\left(B u_{i}\right){ }^{*} z_{i}$ $=v_{i}^{*} B u_{i}=v_{i}^{*} B u_{i}^{*} z_{i}=B u_{i}^{*} v_{i}^{*} z_{i}=B u_{i}^{*} v_{i}$.

Let $E_{i}^{\prime}$ be the conjugate space of $E_{i}$. Then $E_{i}^{\prime}$ is also an $\mathfrak{A} / \Im\left(E_{i}\right)$-module. In fact, if $\Re_{i}$ represents the ring of all endomorphisms of $E_{i}$, then $E_{i}$ is a left $\Re_{i}$-module and $E_{i}^{\prime}$ is a right $\Re_{i}$-module. We shall follow this convention and consider $E_{i}^{\prime}$ as a right $\mathfrak{A} / \Im\left(E_{i}\right)$-module even though $\mathfrak{A} / \Im\left(E_{i}\right)$ is commutative. The bilinear pairing of $E_{i} \times E_{i}^{\prime}$ into $K$ will be written as $u_{i} \times u_{i}^{\prime} \rightarrow\left\langle u_{i}^{\prime}, u_{i}\right\rangle$ so that $\left\langle u_{i}^{\prime} B, u_{i}\right\rangle=\left\langle u_{i}^{\prime}, B u_{i}\right\rangle$ for all $B \in \mathfrak{A} / \Im\left(E_{i}\right)$.

THEOREM 1. Let $x_{j}^{\prime}$ be an arbitrary linear functional on $E_{1} \oplus \cdots \oplus E_{j}$, $j=1, \cdots, t$. Then there exists a unique $f \in S(A)$ such that $\left\langle x_{j}^{\prime}, x_{j}\right\rangle=f\left(x_{j}, z_{j}\right)$ for all $x_{j} \in E_{1} \oplus \cdots \oplus E_{j}, j=1, \cdots, t$.

Proof. The functional $x_{j}^{\prime}$ is uniquely the sum of functionals $x_{1 j}^{\prime}, \cdots, x_{j j}^{\prime}$ on $E_{1}, \cdots, E_{j}$, respectively. If $u_{i}$ and $v_{i}$ are the projections of $u \in E$ and $v \in E$ on $E_{i}$, then the bilinear form $f$ such that

$$
f(u, v)=\sum_{1 \leq j \leq t}\left\langle x_{j j}^{\prime}, \stackrel{u}{u}_{j}^{*} v_{j}\right\rangle+\sum_{1 \leq i<j \leq t}\left\langle x_{i j}^{\prime}, v_{j}^{*} u_{i}+{ }_{u_{j} v_{i}}^{*}\right\rangle
$$

is an element of $\delta(A)$ satisfying the conditions of the theorem. To show uniqueness suppose that $x_{j}^{\prime}=0, j=1, \cdots, t$. Then for any $f \in S(A)$ corresponding to these functionals,

$$
f\left(u_{i}, v_{j}\right)=f\left(u_{i}, v_{j}^{*} z_{j}\right)=f\left(v_{j}^{*} u_{i}, z_{j}\right)=0
$$

when $i \leqq j$, and since $f$ is symmetric it follows that $f=0$.

THEOREM 2. Suppose that $E$ is not cyclic and let $y_{j}^{\prime}$ be an arbitrary linear functional on $E_{1} \oplus \cdots \oplus E_{j-1}, j=2, \cdots, t$. Then there exists a unique 
$f \in Q(A)$ such that $\left\langle y_{j}^{\prime}, y_{j}\right\rangle=f\left(y_{j}, z_{j}\right)$ for all $y_{j} \in E_{1} \oplus \cdots \oplus E_{j-1}, j=2, \cdots, t$.

Proof. The functional $y_{j}^{\prime}$ is uniquely the sum of functionals $y_{1,}^{\prime}, \cdots, y_{j-1, j}^{\prime}$ on $E_{1}, \cdots, E_{j-1}$, and one verifies that the bilinear form $f$ such that

$$
f(u, v)=\sum_{1 \leqq i<j \leq t}\left\langle y_{i j}^{\prime}, v_{j}^{*} u_{i}-u_{j}^{*} v_{i}\right\rangle
$$

is an element of $Q(A)$ satisfying the conditions of the theorem. Uniqueness follows as in the preceding theorem.

Theorem 3. $\mathrm{C}(A)=\mathfrak{S}(A) \oplus_{K} \mathfrak{Q}(A)$.

Proof. If $f \in \mathfrak{e}(A)$ then decompose $f$ into its symmetric and alternating parts, $f=s+a$; the decomposition is unique since char $K \neq 2$. For any $(u, v) \in E \times E$ one obtains $s A(v, u)-s A(u, v)=a A(u, v)+a A(v, u)$, and since the left- and right-hand sides are alternating and symmetric, respectively, it follows that both sides vanish. Hence $s A$ and $a A$ are symmetric and alternating, respectively, so that $s \in \mathcal{S}(A)$ and $a \in Q(A)$ as asserted.

Note that even in the absence of the hypothesis char $K \neq 2, \mathfrak{e}(A)$ is spanned by forms of the types given by $f(u, v)=\left\langle x_{i j}^{\prime}, v_{j}^{*} u_{i}\right\rangle$ and $f(u, v)$ $=\left\langle x_{i j}^{\prime}, u_{j}^{*} v_{i}\right\rangle$.

Corollary 1. $\operatorname{dim}_{K} \delta(A)=\sum_{j=1}^{t}(t-j+1) \operatorname{dim}_{K} E_{j}, \operatorname{dim}_{K} a(A)$ $=\sum_{j=1}^{t}(t-j) \operatorname{dim}_{K} E_{j}$, and $\operatorname{dim}_{K} \mathfrak{e}(A)=\sum_{j=1}^{t}(2 t-2 j+1) \operatorname{dim}_{K} E_{j}$.

THEOREM 4. $S(A)$ contains a nondegenerate form.

Proof. The conjugate spaces $E_{1}^{\prime}, \cdots, E_{t}^{\prime}$ are also cyclic. Let $z_{j}^{\prime}$ generate $E_{j}^{\prime}$ and consider the element $f \in \delta(A)$ given by $f(u, v)=\sum_{1 \leq j \leq t}\left\langle z_{j}^{\prime}, u_{j}^{*} v_{j}\right\rangle$.

Theorem 4 may be used to express the similarity of an arbitrary matrix $(A)$ to its transpose. If $f$ is any nondegenerate form there exists an isomorphism $\bar{f}$ of $E$ onto $E^{\prime}$ such that $\langle\bar{f}(v), u\rangle=f(u, v)$, that is, $\bar{f}$ is the correlation corresponding to $f$. The matrix representations $(f)$ and $(\bar{f})$ of $f$ and $\bar{f}$ are identical and invertible since $f$ is nondegenerate. Furthermore if $f \in \mathcal{S}(A)$ then $(\bar{f})$ and $(\bar{f})(A)$ are symmetric so that $(\bar{f})(A)={ }^{t}(A)^{t}(\bar{f})={ }^{t}(A)(\bar{f})$; that is," '(A) $=(\bar{f})(A)(\bar{f})^{-1}$ as desired.

3. The $\mathfrak{A}$-modules $\mathfrak{C}(A)$ and $\mathfrak{S}(A)$. Let $\Re$ be the ring of all endomorphisms of $E$, and consider $E$ and $E^{\prime}$ as left and right $\Re$-modules, respectively. Let $B \in \Re$ and $C \in \Re$, and let $f$ be an arbitrary bilinear form on $E \times E$. Then for all $(u, v) \in E \times E, \quad(f B) \mathfrak{e}(u, v)=f B(u, C v)=f(u, B C v)=f(B C)(u, v)$, so that $(f B) C=f(B C)$. Thus the $K$-linear space $B$ of all bilinear forms on $E \times E$ is a right $\Re$-module with the composition $f \cdot B=f B$. The following lemma is wellknown.

LEMma 3. If $f$ is any nondegenerate bilinear form on $E \times E$, then the map $B \rightarrow f B$ is an isomorphism of the right $\Re$-module $\Re$ onto the right $\Re$-module $B$. 
Proof. Trivially $B \rightarrow f B$ is a homomorphism of $\Re$ into $B$. Now let $\bar{f}$ represent the correlation $E \rightarrow E^{\prime}$ which corresponds to $f$. If $f B=0$ then $\bar{f} \circ B=0$ hence $B=\bar{f}^{-1} \circ \bar{f} \circ B=0$, so that $B \rightarrow f B$ is an isomorphism. Furthermore for any $g \in B$ we may define $\bar{g}: E \rightarrow E^{\prime}$ by $\langle\bar{g}(v), u\rangle=g(u, v)$ and note that $g(u, v)$ $=\langle\bar{g}(v), \quad u\rangle=\left\langle\bar{f}\left(\bar{f}^{-1} \circ \bar{g}(v)\right), \quad u\right\rangle=f\left(u, \bar{f}^{-1} \circ \bar{g}(v)\right)=f\left(\bar{f}^{-1} \circ \bar{g}\right)(u, v)$; that is, $g=f\left(\bar{f}^{-1} \circ \bar{g}\right)$, so that $B \rightarrow f B$ is onto.

Let $\mathbb{C}(A)$ be the centralizer of $A$ in $\Re$; that is, the ring of all endomorphisms which commute with $A$. If $f \in \mathfrak{C}(A)$, and $B \in \mathbb{E}(A)$ then for all $(u, v) \in E \times E, f B(u, A v)=f(u, B A v)=f(u, A B v)=f(A u, B v)=f B(A u, v)$ so that $\mathrm{C}(A)$ is a right $\mathfrak{S}(A)$-module with the composition $f \cdot B=f B$. Now let $f$ be the nondegenerate element of $s(A)$ given in Theorem 4 and let $\Phi$ denote the corresponding isomorphism of $\Re$ onto $B$ as in Lemma 3 .

Lemma 4. $\Phi$ induces an isomorphism, which will also be denoted $\Phi$, of the right $\mathfrak{E}(A)$-module $\mathfrak{S}(A)$ onto the right $\mathfrak{E}(A)$-module $\mathfrak{e}(A)$.

Proof. Clearly $\Phi$ is a module isomorphism of $\mathfrak{S}(A)$ into $\mathfrak{C}(A)$, by Lemma 3 and the preceding remarks. It remains to show that $f B \in \mathfrak{C}(A)$ implies $B \in \mathbb{C}(A)$; that is, that $\Phi$ is onto. If $f B \in \mathbb{C}(A)$ then $\langle\bar{f}(A B v), u\rangle=f(u, A B v)$ $=f(A u, B v)=f B(A u, v)=f B(u, A v)=f(u, B A v)=\langle\bar{f}(B A v), u\rangle$ for all $(u, v)$ $\in E \times E$, hence $\bar{f}(A B v)=\bar{f}(B A v)$ for all $v \in E$. Since $\bar{f}$ possesses an inverse, $A B v=B A v$ for all $v \in E$, hence $A B=B A$; that is, $B \in \mathbb{E}(A)$ as asserted.

Since $\mathfrak{A} \subset \mathfrak{C}(A), \mathfrak{C}(A)$ and $\mathfrak{C}(A)$ are a fortiori isomorphic as $\mathfrak{A}$-modules. We shall study $\mathfrak{C}(A)$ as an $\mathfrak{A}$-module and use the results to obtain information about $\mathbb{\complement}(A)$.

If $i \leqq j$ let $\mathfrak{C}_{i j}$ denote the class of bilinear forms of the type given by $f(u, v)=\left\langle x_{i j}^{\prime}, v_{j}^{*} u_{i}\right\rangle$, and in particular let $c_{i j}$ be defined by $c_{i j}(u, v)=\left\langle z_{i}^{\prime}, v_{j}^{*} u_{i}\right\rangle$, where $z_{i}^{\prime}$ generates $E_{i}^{\prime}$. Then if $f \in \mathbb{C}_{i j}$ and $B \in \mathfrak{A}$ it follows that $f B(u, v)$ $=f(u, B v)=\left\langle x_{i j}^{\prime},\left(B v_{j}\right) * u_{i}\right\rangle=\left\langle x_{i j}^{\prime}, B v_{j}^{*} u_{i}\right\rangle=\left\langle x_{i j}^{\prime} B, v_{j}^{*} u_{i}\right\rangle$ so that $f B \in \mathbb{C}_{i j}$. Thus $\mathfrak{C}_{i j}$ is a cyclic $\mathfrak{A}$-module which is isomorphic to $E_{i}^{\prime}$, and which is generated by $c_{i j}, i \leqq j$.

Similarly let $\mathfrak{C}_{j i}$ denote the class of bilinear forms of the type given by $f(u, v)=\left\langle x_{i j}^{\prime}, u_{j}^{*} v_{i}\right\rangle$, where $i \leqq j$, and in particular let $c_{j i}$ be defined by $c_{j i}(u, v)$ $=\left\langle z_{i}^{\prime}, u_{j}^{*} v_{i}\right\rangle$. Then $\mathfrak{C}_{j i}$ is a cyclic $\mathfrak{A}$-module which is isomorphic to $E_{i}^{\prime}$ and which is generated by $c_{j i}, i \leqq j$.

The preceding results imply

Theorem 5. $\mathfrak{C}(A)$ and $\mathfrak{S}(A)$ are isomorphic $\mathfrak{A}$-modules, each being the direct sum of $t^{2}$ cyclic $\mathfrak{A}$-modules, $2 t-2 j+1$ of them isomorphic to $E_{j}^{\prime}, j=1, \cdots, t$.

The following corollary is a well-known result of Frobenius.

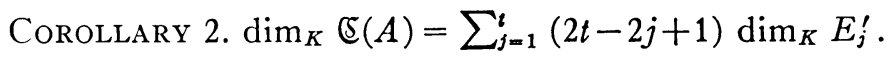

In the sequel we shall let $\mathfrak{E}_{i j} \subset \mathbb{C}(A)$ and $C_{i j} \in \mathfrak{E}_{i j}$ denote the modules and generators, respectively, which are isomorphic to $\mathfrak{e}_{i j} \subset \mathfrak{e}(A)$ and $c_{i j} \in \mathfrak{C}_{i j}$. 
4. An alternate development of the cyclic case. In the cyclic case it is perhaps easier to describe $\mathbb{S}(A)$ directly and then to use Theorem 5 to study $\mathfrak{e}(A)$. We begin with the following well-known result.

Lemma 5. If $E$ is cyclic then $\mathfrak{S}(A)=\mathfrak{A}$.

Proof. For any $B \in \mathbb{E}(A)$ set $u=B z$, where $z$ generates $E$. Then for any $v \in E, B v=B v^{*} z=v^{*} B z=v^{*} u=v^{*} u^{*} z=u^{*} v^{*} z=u^{*} v$, hence $B=u^{*} \in \mathfrak{A}$.

In place of the more exact statement that each $\mathfrak{C}_{i j}$ is an $\mathfrak{A}$-module we need only

Lemma 6. $\delta(A)$ and $Q(A)$ are $\mathfrak{A}$-modules under the composition $f \cdot B=f B$.

Proof. Suppose that $f \in \mathcal{S}(A)$; that is, that $f$ and $f A$ are symmetric. We shall show by induction that $f A^{k} \in \mathcal{S}(A)$ for all $k$. If $f A^{k-1} \in \mathcal{S}(A)$, then $f A^{k}$ is symmetric by the inductive hypothesis, and $\left(f A^{k}\right) A(u, v)=f A^{k}(u, A v)$ $=f A^{k}(A v, u)=f A^{k-1}(A v, A u)$, so that $\left(f A^{k}\right) A$ is also symmetric, as desired. A similar technique may be used to show that $Q(A)$ is an $\mathfrak{A}$-module.

THEоRем 6. If $E$ is cyclic then the $\mathfrak{A}$-modules $\mathfrak{S}(A)$ and $\mathfrak{A}$ are isomorphic, and $\mathfrak{Q}(A)$ is the zero $\mathfrak{P}$-module.

Proof. Let $f$ be that bilinear form such that $f(u, v)=\left\langle z^{\prime}, v^{*} u\right\rangle$, where $z^{\prime}$ generates $E^{\prime}$. Then $f \in \mathcal{S}(A)$, by direct verification, and $f$ is nondegenerate since $f(u, v)=\left\langle z^{\prime} v^{*}, u\right\rangle$, where $u$ and $z^{\prime} v^{*}$ may be arbitrary elements of $E$ and $E^{\prime}$, respectively. Trivially $f B \in \mathcal{S}(A)$ for all $B \in \mathfrak{A}$, and Lemmas 4 and 6 imply that every element of $\mathrm{s}(A)$ is of this form, as before. The second assertion follows from the observation that $\mathfrak{C}(A)=\mathcal{S}(A) \oplus \mathfrak{\imath} \mathfrak{U}(A)$ where both $\mathfrak{C}(A)$ and $\mathfrak{S}(A)$ are isomorphic to $\mathfrak{A}$.

5. The $\mathfrak{A}$-algebra $\mathbb{}(A)$. Since $\mathfrak{A}$ lies in the center of $\mathbb{}(A)$, the latter admits the former as a ring of operators. Briefly, $\mathbb{E}(A)$ is an algebra over the commutative ring $\mathfrak{A}$. Clearly $\mathfrak{S}(A)$ is generated as an $\mathfrak{A}$-algebra by elements which carry one cyclic submodule into another, and such endomorphisms of $E$ are induced in a natural way by the corresponding $\mathfrak{A}$-homomorphisms of one cyclic submodule into another. An $\mathscr{U}$-homomorphism of $E_{j}$ into $E_{i}$ is uniquely determined by assigning the image of any generator $z_{j}$ of $E_{j}$, and it is tempting to conjecture that the image may simply by chosen to be a generator $z_{i}$ of $E_{i}$. Such a conjecture only makes sense when $\Im\left(E_{i}\right) \supseteq \Im\left(E_{j}\right)$, however. This is the case when $\operatorname{dim}_{K} E_{i} \leqq \operatorname{dim}_{K} E_{j}$, and in particular when $i \leqq j$. A little more effort is needed to treat the remaining case.

Let $\rho_{j}$ denote the $j$ th invariant factor of $A$. If $i \leqq j, \rho_{j}=\pi_{j i} \rho_{i}$ for some polynomial $\pi_{j i}$ whose degree is $\operatorname{dim} E_{j}-\operatorname{dim} E_{i}$ and whose leading coefficient is 1 .

Lemma 7. Suppose that $z_{1}, \cdots, z_{t}$ generate $E_{1}, \cdots, E_{t}$, respectively, and that $z_{t}^{\prime}$ generates $E_{t}^{\prime}$. Then there exist unique $z_{1}^{\prime}, \cdots, z_{t-1}^{\prime}$ which generate $E_{1}^{\prime}, \cdots, E_{t-1}^{\prime}$, respectively, and which satisfy $\left\langle z_{j}^{\prime}, B \pi_{j i}(A) z_{j}\right\rangle=\left\langle z_{i}^{\prime}, B z_{i}\right\rangle$ whenever $1 \leqq i \leqq j \leqq t$, for all $B \in \mathfrak{A}$. 
Proof. The uniqueness of $z_{1}^{\prime}, \cdots, z_{t-1}^{\prime}$ is an immediate consequence of the equality $\left\langle z_{i}^{\prime}, B z_{i}\right\rangle=\left\langle z_{t}^{\prime}, B \pi_{t i}(A) z_{t}\right\rangle$. In order to show that $z_{1}^{\prime}, \cdots, z_{t-1}^{\prime}$ exist we first note that the cyclic $\mathfrak{A}$-modules generated by $z_{i}$ and $\pi_{j i}(A) z_{j}$ are $\mathfrak{A}$-isomorphic since each possesses the minimum polynomial $\rho_{\boldsymbol{i}}$. Let $\Gamma_{j i}$ denote the $\mathfrak{A}$-homomorphism of $E_{i}$ into $E_{j}$ induced by setting $\Gamma_{j i}\left(z_{i}\right)=\pi_{j i}(A) z_{j}$. Since $\pi_{k j} \pi_{j i}=\pi_{k i}$ whenever $i \leqq j \leqq k$, it follows that $\Gamma_{k j} \circ \Gamma_{j i}=\Gamma_{k i}$ whenever $i \leqq j \leqq k . \Gamma_{j i}$ induces a dual homomorphism $\Gamma_{\mu}^{\prime}$ of $E_{j}^{\prime}$ into $E_{i}^{\prime}$ which satisfies $\Gamma_{\mathfrak{k}}^{\prime} \circ \Gamma_{k \mathfrak{t}}^{\prime}=\Gamma_{k \mathfrak{k}}^{\prime}$, and for any $B \in \mathfrak{A}$ it follows that

$$
\left\langle\Gamma_{t i}^{\prime}\left(z_{t}^{\prime}\right), B z_{i}\right\rangle=\left\langle\Gamma_{j t}^{\prime}\left(\Gamma_{t j}^{\prime}\left(z_{t}^{\prime}\right)\right), B z_{i}\right\rangle=\left\langle\Gamma_{t j}^{\prime}\left(z_{t}^{\prime}\right), \Gamma_{j i}\left(B z_{i}\right)\right\rangle=\left\langle\Gamma_{t j}^{\prime}\left(z_{t}^{\prime}\right), B \pi_{j i}(A) z_{j}\right\rangle .
$$

Thus $\left\langle z_{i}^{\prime}, B z_{i}\right\rangle=\left\langle z_{j}^{\prime}, B \pi_{j i}(A) z_{j}\right\rangle$ if $z_{j}^{\prime}=\Gamma_{t j}^{\prime}\left(z_{t}^{\prime}\right), j=1, \cdots, t$, and it remains to show that this $z_{j}^{\prime}$ generates $E_{j}^{\prime}$. Let $u_{t}$ be any element of $E_{t}$. Then $\left\langle z_{t}^{\prime} B \pi_{t j}(A), u_{t}\right\rangle=\left\langle z_{t}^{\prime}, B u_{t}^{*} \pi_{t j}(A) z_{i}\right\rangle=\left\langle z_{j}^{\prime}, B u_{t}^{*} z_{j}\right\rangle=\left\langle z_{j}^{\prime} B, u_{t}^{*} z_{j}\right\rangle$, so that if $\sigma_{j}$ is a polynomial of minimum degree such that $z_{j}^{\prime} \sigma_{j}(A)=0$ it follows that $z_{t}^{\prime} \sigma_{j}(A) \pi_{t j}(A)=0$. But this implies that degree $\sigma_{j} \pi_{t j} \geqq \operatorname{dim} E_{t}^{\prime}$, since $E_{t}^{\prime}$ is cyclic, hence that degree $\sigma_{j}=$ degree $\sigma_{j} \pi_{i j}-$ degree $\pi_{t j} \geqq \operatorname{dim} E_{j}^{\prime}$. The opposite inequality, degree $\sigma_{j} \leqq$ degree $\rho_{j}=\operatorname{dim} E_{j}^{\prime}$, is a trivial consequence of the fact that $E_{j}^{\prime}$ is cyclic. Hence degree $\sigma_{j}=\operatorname{dim} E_{j}^{\prime}$ so that $\left(z_{j}^{\prime}, z_{j}^{\prime} A, \cdots\right.$, $\left.z_{j}^{\prime} A^{\operatorname{dim} E_{j}^{\prime-1}}\right)$ is a basis of $E_{j}^{\prime}$; that is, $z_{j}^{\prime}$ generates $E_{j}^{\prime}$ as asserted.

We shall say that a set $\left(z_{1}^{\prime}, \cdots, z_{t}^{\prime}\right)$ of generators of $E_{1}^{\prime}, \cdots, E_{t}^{\prime}$ is coherently related to a set $\left(z_{1}, \cdots, z_{t}\right)$ of generators of $E_{1}, \cdots, E_{t}$ if it satisfies the conditions of Lemma 7 . Clearly $\left(z_{1}^{\prime}, \cdots, z_{t}^{\prime}\right)$ is coherently related to $\left(z_{1}, \cdots, z_{t}\right)$ if and only if $\left(z_{1}, \cdots, z_{t}\right)$ is coherently related to $\left(z_{1}^{\prime}, \cdots, z_{t}^{\prime}\right)$.

We return to the problem of describing the $\mathfrak{A}$-algebra $\mathfrak{S}(A)$. If $i \leqq j$ let $\Gamma_{j i}$ denote the $\mathfrak{A}$-homomorphism $E_{i} \rightarrow E_{j}$ generated by $\Gamma_{j i}\left(z_{i}\right)=\pi_{j i}(A) z_{j}$, as in Lemma 7 , and let $\Gamma_{i j}$ denote the $\mathfrak{A}$-homomorphism $E_{j} \rightarrow E_{i}$ generated by $\Gamma_{i j}\left(z_{j}\right)=z_{i}$. For the convenience of the notation we define $\pi_{i j}$ in the case $i \leqq j$ by setting $\pi_{i j}(\lambda)=1$. Then $\Gamma_{j i}\left(z_{i}\right)=\pi_{j i}(A) z_{j}$ for all indices $i, j=1, \cdots, t$.

Let $C_{i j}$ denote the endomorphism of $E$ which is induced by $\Gamma_{i j}$; that is, if $v_{j}$ is the projection of any $v \in E$ in $E_{j}$, then $C_{i j} v=v_{j}^{*} \pi_{i j}(A) z_{i}, i, j=1, \cdots, t$. We shall show that $\Phi\left(C_{i j}\right)=c_{i j}$ as indicated at the end of $\S 3$, where $\Phi$ is the isomorphism defined in Lemma 4 . If $\left(z_{1}, \cdots, z_{t}\right)$ and $\left(z_{1}^{\prime}, \cdots, z_{t}^{\prime}\right)$ are coherently related, and if $f$ is the nondegenerate element of $\delta(A)$ given in Theorem 4 , then for $i \leqq j$ we have $f C_{i j}(u, v)=f\left(u, C_{i j} v\right)=f\left(u, v_{j}^{*} \pi_{i j}(A) z_{i}\right)$ $=f\left(u, v_{j}^{*} z_{i}\right)=\left\langle z_{i}^{\prime}, u_{i}^{*} v_{j}^{*} z_{i}\right\rangle=c_{i j}(u, v)$, since $\pi_{i j}(A)$ is the identity, and $f C_{j i}(u, v)$ $=f\left(u, v_{i}^{*} \pi_{j i}(A) z_{j}\right)=\left\langle z_{j}^{\prime}, u_{j}^{*} v_{i}^{*} \pi_{j i}(A) z_{j}\right\rangle=\left\langle z_{i}^{\prime}, u_{j}^{*} v_{i}^{*} z_{i}\right\rangle=c_{j i}(u, v)$, since $\left(z_{1}, \cdots, z_{t}\right)$ and $\left(z_{1}^{\prime}, \cdots, z_{t}^{\prime}\right)$ are coherently related. Thus $f C_{i j}=c_{i j}$ for all values of $i$ and $j$, as asserted.

In order to describe $\mathfrak{S}(A)$ as an $\mathfrak{A}$-algebra it suffices to display a multiplication table of the $C_{i j}$ 's. Since $C_{i j} z_{k}=\delta_{j k} \pi_{i j}(A) z_{i}$ for all $i, j, k=1, \cdots, t$, where $\delta_{j k}$ is the Kronecker $\delta$, one easily verifies that 
THEOREM 7.

$$
C_{i j} C_{k l}=\left\{\begin{array}{l}
\delta_{j k} \pi_{i j}(A) \pi_{k l}(A) C_{i l} \text { if } i \leqq l, \\
\delta_{j k} \pi_{l k}(A) \pi_{j i}(A) C_{i l} \text { if } i \geqq l .
\end{array}\right.
$$

Incidentally, it should be noted that $\pi_{k j} \pi_{j i}=\pi_{k i}$ if and only if $i \leqq j \leqq k$ or $i \geqq j \geqq k$, so that in general this result cannot be simplified further.

It is to be expected that the $\mathscr{A}$-submodules of $\mathbb{E}(A)$ which correspond to $\mathfrak{S}(A)$ and $\mathfrak{Q}(A)$ might have special properties as $\mathfrak{A}$-algebras. If $\mathfrak{S}(A)$ $=\Phi^{-1}(\mathcal{S}(A))$ and $\mathfrak{R}(A)=\Phi^{-1}(\mathscr{Q}(A))$, then it is clear that the $\mathfrak{H}$-modules $\mathfrak{S}(A)$ and $\mathfrak{R}(A)$ are generated by elements of the types $C_{i j}+C_{j i}$ and $C_{i j}-C_{j i}$ respectively.

Define Jordan and Lie multiplication by $\{B, C\}=B C+C B$ and $[B, C]$ $=B C-C B$, respectively. Then if $f, f B$, and $f C$ are symmetric,

$$
\begin{aligned}
f B C(u, v) & =f B(u, C v)=f B(C v, u)=f(C v, B u)=f(B u, C v) \\
& =f C(B u, v)=f C(v, B u)=f C B(v, u),
\end{aligned}
$$

so that $f(B C+C B)$ is also symmetric. Hence $\subseteq(A)$ is closed under Jordan multiplication, and in a similar fashion $\mathfrak{R}(A)$ is closed under Lie multiplication.

Thus, since $\mathfrak{S}(A)$ and $\mathfrak{R}(A)$ are the direct sums of the cyclic $\mathfrak{A}$-modules generated by $C_{i j}+C_{j i}$ and $C_{i j}-C_{j i}$, respectively, we obtain

THEOREM 8. $\mathfrak{S}(A)=\mathfrak{S}(A) \oplus \mathfrak{M}(A)$ where $\mathfrak{S}(A)$ is closed under Jordan multiplication and $\mathfrak{R}(A)$ is closed under Lie multiplication. Furthermore $\mathfrak{S}(A)$ is the direct sum of $t(t+1) / 2$ cyclic $\mathfrak{A}$-modules, $t-i+1$ of which are isomorphic to $E_{i}^{\prime}, i=1, \cdots, t$, and $\mathfrak{R}(A)$ is the direct sum of $t(t-1) / 2$ cyclic $\mathfrak{A}$-modules, $t-i$ of which are isomorphic to $E_{i}^{\prime}, i=1, \cdots, t-1$.

Since elements of the types $C_{i j}+C_{j i}$ and $C_{i j}-C_{j i}$ generate $\mathfrak{S}(A)$ and $\mathfrak{L}(A)$, respectively, the appropriate multiplication tables may be computed directly from Theorem 7 .

6. Matrix representations of $\mathfrak{e}(A)$ in the cyclic case. If $E$ is cyclic, with a generator $z$, then $\left(z, A z, \cdots, A^{n-1} z\right)$ will be called a left canonical basis of $E$. If $z^{\prime}$ generates $E^{\prime}$, then the basis $\left(u_{1}, \cdots, u_{n}\right)$ of $E$ which is the dual of the basis $\left(z^{\prime}, z^{\prime} A, \cdots, z^{\prime} A^{n-1}\right)$ of $E^{\prime}$ will be called a right canonical basis of $E$. If the elements of $E$ are regarded as column vectors, then the matrices $(A)_{l}$ and $(A)_{r}$ which represent $A$ with respect to left and right canonical bases, are companion matrices with 1 's below and above the main diagonal, respectively. For convenience we shall simply refer to $(A)_{l}$ and $(A)_{r}$ as left and right representations of $A$. If $f$ is any bilinear form $f$ on $E \times E$, we define $(f)_{l}$ and $(f)_{r}$ in a similar fashion, these being the left and right representations of $f$. Clearly $(A)_{r}={ }^{t}(A)_{l}$, where ${ }^{t}(A)_{l}$ is the transpose of $(A)_{l}$. 
Since $E$ is cyclic, $\mathfrak{A}=\mathfrak{S}(A)$ according to Lemma 5 , so that the matrix representations of $\mathfrak{S}(A)$ with respect to any basis may be obtained from the powers of the representation of $A$. The representations of $\mathcal{C}(A)$ are not so trivial, however, and will be developed in this section.

We begin with the left representation of $\mathfrak{e}(A)$.

Let $\alpha$ represent the characteristic polynomial of $A, \alpha(\lambda)=\alpha_{0}+\alpha_{1} \lambda+\ldots$ $+\alpha_{n-1} \lambda^{n-1}-\lambda^{n}$, and let $(A]$ represent the $n \times(2 n-1)$ matrix whose $k$ th column is the first column of $(A)_{l}^{k-1}$. Thus if $n=3$,

$$
(A]=\left(\begin{array}{lllll}
1 & 0 & 0 & \alpha_{0} & \alpha_{2} \alpha_{0} \\
0 & 1 & 0 & \alpha_{1} & \alpha_{0}+\alpha_{2} \alpha_{1} \\
0 & 0 & 1 & \alpha_{2} & \alpha_{1}+\alpha_{2}^{2}
\end{array}\right)
$$

It should be noted that $(A]$ may be computed in terms of the first $n-1$ powers of $(A)_{l}$.

Any matrix $\left(a_{i j}\right)$ such that $a_{i j}$ depends only on $i+j$ is called a Hankel matrix, and such a matrix is uniquely defined by assigning elements of $K$ to each of $2 n-1$ values of $i+j, i+j=2, \cdots, 2 n$.

TheOREM 9. If $E$ is cyclic, then the $n$ Hankel matrices corresponding to the $n$ rows of $(A]$ constitute a basis of the left representation of $\mathrm{e}(A)$.

Thus if $n=3$ the basis is

$$
\left(\begin{array}{lll}
1 & 0 & 0 \\
0 & 0 & \alpha_{0} \\
0 & \alpha_{2} & \alpha_{2} \alpha_{0}
\end{array}\right), \quad\left(\begin{array}{lll}
0 & 1 & 0 \\
1 & 0 & \alpha_{1} \\
0 & \alpha_{1} & \alpha_{0}+\alpha_{2} \alpha_{1}
\end{array}\right), \quad\left(\begin{array}{lll}
0 & 0 & 1 \\
0 & 1 & \alpha_{2} \\
1 & \alpha_{2} & \alpha_{1}+\alpha_{2}^{2}
\end{array}\right),
$$

and one easily checks that multiplication on the right by $(A)_{l}$ yields linear combinations of these matrices. In fact, these matrices correspond to the basis elements $(1,0,0),(0,1,0),(0,0,1)$ of the right $\mathfrak{A}$-module $E^{\prime}$.

Proof. Let $\left(u_{1}, \cdots, u_{n}\right)$ represent the left canonical basis $\left(z, \cdots, A^{n-1} z\right)$ of $E$, and let $\left(u_{1}^{\prime}, \cdots, u_{n}^{\prime}\right)$ be the dual basis of $E^{\prime}$. Let $f_{k}$ be that member of $\mathfrak{e}(A)$ whose values are given by $f_{k}(u, v)=\left\langle u_{k}^{\prime}, u^{*} v\right\rangle$. Then $f_{1}, \cdots, f_{n}$ span $\mathfrak{C}(A)$ as a vector space over $K$, and the element in the $i$ th row and $j$ th column of $\left(f_{k}\right)_{l}$ is $f_{k}\left(u_{i}, u_{j}\right)=\left\langle u_{k}^{\prime}, A^{i+j-2} z\right\rangle$, which is the element in the $k$ th row and $(i+j-1)$ st column of $(A]$, as asserted.

Note that $f_{n}$ is nondegenerate, so that we might also take the matrices $\left(f_{n}\right)_{l},\left(f_{n}\right)_{l}(A)_{l}, \cdots,\left(f_{n}\right)_{l}(A)_{l}^{n-1}$ as a basis of the left representation of $\mathbb{e}(A)$. These matrices correspond to the elements of a left canonical basis of the left $\mathfrak{A}$-module $E$.

For convenience we write $\alpha_{n}=-1$ and $\alpha_{j}=0$ if $j<0$ or $j>n$. Let $[A)$ represent the $(2 n-1) \times(n-1)$ matrix whose $k$ th column contains the entries $\alpha_{1-k}, \cdots, \alpha_{2 n-k-1}$. Thus if $n=3$, 


$$
[A)=\left(\begin{array}{rr}
\alpha_{0} & 0 \\
\alpha_{1} & \alpha_{0} \\
\alpha_{2} & \alpha_{1} \\
-1 & \alpha_{2} \\
0 & -1
\end{array}\right)
$$

CoRollary 3. Let (w) be any row vector with $2 n-1$ entries. Then the Hankel matrix corresponding to the entries of $(w)$ is a left representation of an element of $\mathrm{e}(A)$ if and only if $(w)[A)=0$.

Proof. The Cayley-Hamilton theorem implies $\sum_{k=0}^{n} \alpha_{k}\left\langle u_{i}^{\prime}, A^{k+j-1} z\right\rangle=0$, $j=1, \cdots, n-1$, so that $(A][A)=0$, and the result follows from the observation that $[A)$ is of rank $n-1$.

Now suppose that $\left(u_{1}, \cdots, u_{n}\right)$ is a right canonical basis and set $u_{0}=0$.

Lemma 8. $A u_{i}=u_{i-1}+\alpha_{i-1} u_{n}, i=1, \cdots, n$.

Proof. If $j\left\langle n\right.$ then $\left\langle z^{\prime} A^{j-1}, A u_{i}\right\rangle=\left\langle z^{\prime} A^{i}, u^{i}\right\rangle=\delta_{j+1, i}=\delta_{j, i-1}=\left\langle z^{\prime} A^{j-1}, u_{i-1}\right\rangle$, and if $j=n$ then

$$
\left\langle z^{\prime} A^{i-1}, A u_{i}\right\rangle=\left\langle z^{\prime} A^{n}, u_{i}\right\rangle=\left\langle z^{\prime} \sum_{k=0}^{n-1} \alpha_{k} A^{k}, u_{i}\right\rangle=\alpha_{i-1}=\left\langle z^{\prime} A^{j-1}, \alpha_{i-1} u_{n}\right\rangle .
$$

Consequently $u_{n}$ generates $E$, and we may define $u_{i}^{*} \in \mathfrak{A}$ by setting $u_{i}=u_{i}^{*} u_{n}$.

LEMma 9.

$$
A^{k-1} u_{i}^{*}= \begin{cases}-\sum_{j=i}^{n} \alpha_{j} A^{j-i+k-1} & \text { if } 0<k \leqq i \\ +\sum_{j=0}^{i-1} \alpha_{j} A^{j-i+k-1} & \text { if } i<k \leqq n\end{cases}
$$

Proof. Since $u_{n}^{*}$ is the identity $I$ on $E$, Lemma 8 implies that $u_{i-1}^{*}=A u_{i}^{*}$ $-\alpha_{i-1} I$, and one may proceed by induction on $n-i$ to prove that $u_{i}^{*}$ $=\sum_{j=i}^{n} \alpha_{j} A^{j-i}$. If $0<k \leqq i$, the first result is obtained upon multiplication by $A^{k-1}$. If $i<k \leqq n$, then $A^{k-1} u_{i}^{*}=A^{-i+k-1}\left(A^{i} u_{i}^{*}\right)=A^{-i+k-1}\left(-\sum_{j=\imath}^{n} \alpha_{j} A^{j}\right)$ $=A^{-i+k-1}\left(+\sum_{j=0}^{i-1} \alpha_{j} A^{j}\right)$, as asserted since $\sum_{j=0}^{i-1} \alpha_{j} A^{j}+\sum_{j=1}^{n} \alpha_{j} A^{j}=0$ by the Cayley-Hamilton theorem.

In order to obtain a basis of the right representations of $\mathfrak{e}(A)$, we let $f$ be the nondegenerate form which is defined in Theorem 4 . In the present case $f$ is given by $f(u, v)=\left\langle z^{\prime}, u^{*} v\right\rangle$, where $u=u^{*} u_{n}$. Then $f, f A, \cdots, f A^{n-1}$ span $\mathfrak{C}(A)$ as a vector space over $K$, and we need only to compute $f A^{k-1}\left(u_{i}, u_{j}\right)$, $i, j, k=1, \cdots, n$. 
THEOREM 10. If $E$ is cyclic, and if $\left(u_{1}, \cdots, u_{n}\right)$ is a right canonical basis of $E$, then

$$
f A^{k-1}\left(u_{i}, u_{j}\right)=\left\{\begin{array}{cl}
-a_{i+j-k} & \text { if } 0<k \leqq i, 0<k \leqq j \\
+a_{i+j-k} & \text { if } i<k \leqq n, j<k \leqq n \\
0 & \text { otherwise. }
\end{array}\right.
$$

Thus if $n=3$ the right representations $(f)_{r},(f A)_{r},\left(f A^{2}\right)_{r}$ are

$$
\left(\begin{array}{ccc}
-\alpha_{1} & -\alpha_{2} & 1 \\
-\alpha_{2} & 1 & 0 \\
1 & 0 & 0
\end{array}\right), \quad\left(\begin{array}{ccc}
\alpha_{0} & 0 & 0 \\
0 & -\alpha_{2} & 1 \\
0 & 1 & 0
\end{array}\right), \quad\left(\begin{array}{lll}
0 & \alpha_{0} & 0 \\
\alpha_{0} & \alpha_{1} & 0 \\
0 & 0 & 1
\end{array}\right),
$$

and one easily checks that multiplication on the right by $(A)_{r}$ yields linear combinations of these matrices. In fact, these matrices correspond to the basis elements $(1,0,0),(0,1,0),(0,0,1)$ of the right $\mathfrak{A}$-module $E^{\prime}$.

Proof. Since $f A^{k-1}\left(u_{i}, u_{j}\right)=\left\langle z^{\prime} A^{k-1} u_{i}^{*}, u_{j}\right\rangle$, we may apply Lemma 9. If $0<k \leqq i$ then

$$
f A^{k-1}\left(u_{i}, u_{j}\right)=-\sum_{h=i}^{n} \alpha_{h}\left\langle z^{\prime} A^{h-i+k-1}, u_{j}\right\rangle=\left\{\begin{array}{cl}
-\alpha_{i+j-k} & \text { if } 0<k \leqq j \\
0 & \text { otherwise }
\end{array}\right.
$$

and similarly if $i<k \leqq n$ then

$$
f A^{k-1}\left(u_{i}, u_{j}\right)=\left\{\begin{array}{cl}
+\alpha_{i+j-k} & \text { if } j<k \leqq n \\
0 & \text { otherwise }
\end{array}\right.
$$

The right representation has the advantage that its entries are linear and homogeneous in the coefficients $\alpha_{0}, \cdots, \alpha_{n}$ of the characteristic polynomial of $A$.

Note that $\left(f_{n}\right)_{l}$ and $(f)_{r}$ are inverse matrices. This is an immediate consequence of the fact that if a left canonical basis of $E$ is employed, then any representation of a bilinear form on $E \times E$ with respect to a right canonical basis may be considered as a bilinear form on $E^{\prime} \times E^{\prime}$.

7. Matrix representations of $\mathbb{E}(A)$ in the general case. Suppose that $z_{1}, \cdots, z_{t}$ generate $E_{1}, \cdots, E_{t}$, respectively. Then, if $n_{j}=\operatorname{dim} E_{j}$,

$$
\left(z_{1}, A z_{1}, \cdots, A^{n_{1}-1} z_{1} ; \cdots ; z_{t}, A z_{t}, \cdots, A^{n_{t-1}} z_{t}\right)
$$

is a basis of $E$ which we shall call a left canonical basis of $E$. Let $\left(u_{1,1}^{\prime}, \cdots\right.$, $\left.u_{1, n_{1}}^{\prime} ; \cdots ; u_{t, 1}^{\prime}, \cdots, u_{t, n_{t}}^{\prime}\right)$ denote the dual basis, and note that $u_{j, n_{j}}^{\prime}$ generates $E_{j}^{\prime}$, by an obvious generalization of Lemma 8 to the ring $\mathfrak{A} / \mathfrak{I}\left(E_{j}\right)$, $j=1, \cdots, t$.

The following lemma will not be used explicitly in the sequel. It is of crucial importance in establishing the matrix correspondence of elements in a 
left representation of $\mathfrak{C}(A)$ with the representations of their images in $\mathfrak{C}(A)$ under the $\mathfrak{A}$-isomorphism $\Phi$, however, and is presented here for this reason.

Lemma $10 .\left(z_{1}, \cdots, z_{t}\right)$ and $\left(u_{1, n_{1}}^{\prime}, \cdots, u_{t, n_{t}}^{\prime}\right)$ are coherently related.

Proof. It suffices to show that if $i \leqq j$ then

$$
\left\langle u_{i, n_{i}}^{\prime}, A^{k-1} z_{i}\right\rangle=\left\langle u_{j, n_{j}}^{\prime}, A^{k-1} \pi_{j i}(A) z_{j}\right\rangle, \quad k=1, \cdots, n_{i} .
$$

But since $\left(z_{i}, \cdots, A^{n_{i}-1} z_{i}\right)$ and $\left(u_{i, 1}^{\prime}, \cdots, u_{i, n_{i}}^{\prime}\right)$ are dual bases of $E_{i}$ and $E_{i}^{\prime}$ it follows that $\left\langle u_{i, n_{i}}^{\prime}, A^{k-1} z_{i}\right\rangle=\delta_{k, n_{i}}$. Similarly since $\left(z_{j}, \cdots, A^{n_{j}-1} z_{j}\right)$ and $\left(u_{j}^{\prime}, \cdots, u_{j, n_{j}}^{\prime}\right)$ are dual bases of $E_{j}$ and $E_{j}^{\prime}$, and since the leading coefficient of $\pi_{j i}$ is 1 , it follows that $\left\langle u_{j, n_{j}}^{\prime}, A^{k-1} \pi_{j i}(A) z_{j}\right\rangle=\delta_{k, n_{i}}$.

Suppose that $z_{1}^{\prime}, \cdots, z_{t}^{\prime}$ are arbitrarily chosen generators of $E_{1}^{\prime}, \cdots, E_{t}^{\prime}$, respectively. Then $\left(z_{1}^{\prime}, z_{1}^{\prime} A, \cdots, z_{1}^{\prime} A^{n_{1}-1} ; \cdots ; z_{t}^{\prime}, z_{t}^{\prime} A, \cdots, z_{t}^{\prime} A^{n_{t}-1}\right)$ is a basis of $E^{\prime}$, and its dual basis $\left(u_{1,1}, \cdots, u_{1, n_{1}} ; \cdots ; u_{t, 1}, \cdots, u_{t, n_{t}}\right)$ will be called a right canonical basis of $E$. As in the preceding case, $u_{j, n_{j}}$ generates $E_{j}$, and $\left(z_{1}^{\prime}, \cdots, z_{t}^{\prime}\right)$ and $\left(u_{1, n_{1}}, \cdots, u_{t, n_{t}}\right)$ are coherently related.

In general $\mathfrak{S}(A) \neq \mathfrak{A}$ so that the representations of $\mathfrak{S}(A)$ can no longer be obtained in terms of the representations of powers of $A$. However, since $\mathfrak{S}(A)$ is the direct sum of the cyclic $\mathfrak{A}$-modules $\mathfrak{夭}_{i j}$ generated by $C_{i j}, i, j$ $=1, \cdots, t$, it will suffice to obtain matrix representations of $C_{i j}$. Furthermore, since $C_{i j}$ takes $E_{j}$ into $E_{i}$ and vanishes outside of $E_{j}$, we shall consider only the corresponding homomorphism $\Gamma_{i j}$ of $E_{j}$ into $E_{i}$.

Let $A_{i}$ and $A_{j}$ denote the restrictions of $A$ to $E_{i}$ and $E_{j}$, respectively. Then since $A C_{i j}=C_{i j} A$, it follows that $A_{i} \Gamma_{i j}=\Gamma_{i j} A_{j}$. Thus we may represent $\mathfrak{S}_{i j}$ in terms of the representations of $\Gamma_{i j}, A_{i} \Gamma_{i j}, \cdots, A_{i}^{n_{i}-1} \Gamma_{i j}$ if $i \leqq j$, and in terms of the representations of $\Gamma_{i j}, \Gamma_{i j} A_{j}, \cdots, \Gamma_{i j} A_{j}^{n_{j}-1}$ if $j \leqq i$. Since $E_{i}$ and $E_{j}$ are cyclic modules for the rings generated by $A_{i}$ and $A_{j}$, it remains to find the representations of $\Gamma_{i j}$ in the two cases $i \leqq j$ and $i \geqq j$. Furthermore, since the dual of a left canonical basis is a right canonical basis, the right representation $\left(\Gamma_{i j}\right)_{r}$ of $\Gamma_{i j}$ is simply the transpose of the left representation $\left(\Gamma_{j i}\right)_{l}$ of $\Gamma_{j i}$. Thus it will suffice to find $\left(\Gamma_{i j}\right)_{l}$ in the two cases $i \leqq j$ and $i \geqq j$.

We shall always take $i \leqq j$ in the sequel, so that $\Gamma_{i j}$ and $\Gamma_{j i}$ will be understood to be the two distinct cases. For convenience we write $r, s, \rho, \pi$ in place of $n_{i}, n_{j}, \rho_{i}, \pi_{j i}$, respectively, where $\rho$ is the $i$ th invariant factor, $\rho(\lambda)$ $=\rho_{0}+\rho_{1} \lambda+\cdots+\rho_{r-1} \lambda^{r-1}-\lambda^{r}$, and where $\pi(\lambda)=\pi_{0}+\pi_{1} \lambda+\cdots$ $+\pi_{s-r-1} \lambda^{s-r-1}+\lambda^{s-r}$. Also we set $\rho_{r}=-1, \pi_{s-r}=+1$, and $\pi_{j}=0$ if $j<0$ or $j>s-r$.

Recall that the elements of $E$ are taken as column vectors, so that if $r=2$,

$$
\left(A_{i}\right)_{l}=\left(\begin{array}{ll}
0 & \rho_{0} \\
1 & \rho_{1}
\end{array}\right) \text { and }\left(A_{i}\right)_{r}=\left(\begin{array}{cc}
0 & 1 \\
\rho_{0} & \rho_{1}
\end{array}\right), \text { e.g. }
$$

Let $\left[\Gamma_{i j}\right]$ represent the $r \times s$ matrix whose $h$ th column is the first column of $\left(A_{i}\right)_{l}^{h-1}$. Thus if $r=2$ and $s=3$, 


$$
\left[\Gamma_{i j}\right]=\left(\begin{array}{ccc}
1 & 0 & \rho_{0} \\
0 & 1 & \rho_{1}
\end{array}\right) .
$$

Lemma 11. If $i \leqq j$ then $\left(\Gamma_{i j}\right)_{l}=\left[\Gamma_{i j}\right]$.

Proof. Since $\Gamma_{i j}\left(A^{h-1} z_{j}\right)=A^{h-1} z_{i}$, the element in the $k$ th row and $h$ th column of $\left(\Gamma_{i j}\right)_{l}$ is $\left\langle u_{i, k}^{\prime}, A^{h-1} z_{i}\right\rangle$, which is precisely the element in the $k$ th row and $h$ th column of $\left[\Gamma_{i j}\right], k=1, \cdots, r, h=1, \cdots, s$.

One easily verifies that $\left(\Gamma_{i j}\right)_{l}$ is of rank $r$ and that $\left(A_{i}\right)_{l}\left(\Gamma_{i j}\right)_{l}=\left(\Gamma_{i j}\right)_{l}\left(A_{j}\right)_{l}$ as desired.

Let $\left[\Gamma_{j i}\right]$ denote the $s \times r$ matrix whose $k$ th column contains the entries $\pi_{1-k}, \cdots, \pi_{s-k}$. Thus if $r=2$ and $s=3$,

$$
\left[\Gamma_{j i}\right]=\left(\begin{array}{ll}
\pi_{0} & 0 \\
1 & \pi_{0} \\
0 & 1
\end{array}\right) .
$$

Lemma 12. If $i \leqq j$ then $\left(\Gamma_{i}\right)_{l}=\left[\Gamma_{j i}\right]$.

Proof. Since $\Gamma_{j i}\left(A^{k-1} z_{i}\right)=A^{k-1} \pi(A) z_{j}$, the element in the $h$ th row and $k$ th column of $\left(\Gamma_{j i}\right)_{l}$ is $\left\langle u_{j, h}^{\prime}, A^{k-1} \pi(A) z_{j}\right\rangle$, which is precisely the element in the $h$ th row and $k$ th column of $\left[\Gamma_{j i}\right], k=1, \cdots, r, h=1, \cdots, s$.

Summarizing, we have

THEOREM 11. If $i \leqq j$ then the left representation of $\mathfrak{C}_{i j}$ consists of zeros except in an $r \times s$ block on or above the diagonal, which contains linear combinations of $\left(A_{i}\right)_{l}^{k-1}\left[\Gamma_{i j}\right], k=1, \cdots, r$, and the left representation of $\mathfrak{夭}_{j i}$ consists of zeros except in an $s \times r$ block on or below the diagonal, which contains linear combinations of $\left[\Gamma_{j i}\right]\left(A_{i}\right)_{l}^{k-1}, k=1, \cdots, r$.

8. Matrix representations of $\mathfrak{C}(A)$ in the general case. Since $\mathbb{E}(A)$ is the direct sum of the $\mathfrak{A}$-modules $\mathfrak{C}_{i j}$ we shall find the matrix representations of the latter modules. Each $\mathcal{C}_{i j}$ is generated by a bilinear form $c_{i j}$ on $E \times E$ which vanishes except on $E_{i} \times E_{j}$, so that it will suffice to consider the restriction $\gamma_{i j}$ of $c_{i j}$ to $E_{i} \times E_{j}$. If $i \leqq j$, then if $u \in E_{i}$ and $v \in E_{j}, \gamma_{i j}(u, v)=\left\langle z_{i}^{\prime}, v^{*} u\right\rangle$ by definition. Since any representation $\left(c_{j i}\right)$ of $c_{j i}$ is the transpose of the corresponding representation of $c_{i j}$, we do not need to consider the case $i>j$.

Let $f$ be the nondegenerate element of $\mathfrak{e}(A)$ given in Theorem 4 . Then $c_{i j}=f C_{i j}$ provided that one chooses coherently related sets of generators of $E_{1}, \cdots, E_{\imath}$ and $E_{1}^{\prime}, \cdots, E_{t}^{\prime}$ in defining $f$. If $\gamma$ represents the restriction of $f$ to $E_{i} \times E_{i}$, it follows that $\gamma_{i j}=\gamma \Gamma_{i j}$, and since $E_{i}$ is cyclic we may use Theorems 9 and 10 to obtain $(\gamma)_{l}$ and $(\gamma)_{r}$. The following two theorems are immediate consequences of these results.

THEOREM 12. Let $i \leqq j$ and let $\left(\gamma_{k}\right)_{l}$ be the kth element of the basis of the left canonical representation of $\mathrm{e}\left(A_{i}\right)$, as in Theorem 9 . Then the left representation 
of $\mathfrak{C}_{i j}$ consists of zeros except in an $r \times s$ block on or above the diagonal, which contains linear combinations of $\left(\gamma_{1}\right)_{l}\left(\Gamma_{i j}\right)_{l}, \cdots,\left(\gamma_{r}\right)_{l}\left(\Gamma_{i j}\right)_{l}$. The left representation of $\mathfrak{C}_{j i}$ is the transpose of this.

Thus if $r=2$ and $s=3$ the nonzero block above the diagonal consists of linear combinations of

$$
\left(\begin{array}{ll}
1 & 0 \\
0 & \rho_{0}
\end{array}\right)\left(\begin{array}{lll}
1 & 0 & \rho_{0} \\
0 & 1 & \rho_{1}
\end{array}\right) \text { and }\left(\begin{array}{cc}
0 & 1 \\
1 & \rho_{1}
\end{array}\right)\left(\begin{array}{lll}
1 & 0 & \rho_{0} \\
0 & 1 & \rho_{1}
\end{array}\right)
$$

It should be noted that if the indicated multiplications are performed one obtains further Hankel matrices, as in Theorem 9. In fact, one may even prove Theorem 12 directly by using the technique of Theorem 9.

Theorem 13. Let $i \leqq j$ and let $\left(\gamma A^{k-1}\right)_{r}$ be the kth element of the basis of the right canonical representation of $\mathrm{e}\left(A_{i}\right)$, as in Theorem 10 . Then the right representation of $\mathfrak{C}_{i j}$ consists of zeros except in an $r \times s$ block on or above the diagonal, which contains linear combinations of $(\gamma)_{r}\left(\Gamma_{i j}\right)_{r}, \cdots,\left(\gamma A_{i}^{r-1}\right)_{r}\left(\Gamma_{i j}\right)_{r}$. The right representation of $\mathfrak{C}_{j i}$ is the transpose of this.

In order to compute $\left(\Gamma_{i j}\right)_{r}$ we note that $\left(\Gamma_{i j}\right)_{r}={ }^{t}\left(\Gamma_{j i}\right)_{l}={ }^{t}\left[\Gamma_{j i}\right]$. Thus if $r=2$ and $s=3$ the nonzero block above the diagonal consists of linear combinations of

$$
\left(\begin{array}{cc}
-\rho_{1} & 1 \\
1 & 0
\end{array}\right)\left(\begin{array}{lll}
\pi_{0} & 1 & 0 \\
0 & \pi_{0} & 1
\end{array}\right) \text { and }\left(\begin{array}{ll}
\rho_{0} & 0 \\
0 & 1
\end{array}\right)\left(\begin{array}{lll}
\pi_{0} & 1 & 0 \\
0 & \pi_{0} & 1
\end{array}\right) \text {. }
$$

9. Remarks on the nondegenerate forms in $\delta(A)$. Suppose that $A$ is cyclic and that $z$ generates $E$. Then the $n$th element of the dual of the left canonical basis $\left(z, A z, \cdots, A^{n-1} z\right)$ generated by $z$ is an $E^{\prime}$-generator $z^{\prime}$, by the dual of Lemma 8. Similarly the $n$th element of the dual basis of $\left(z^{\prime}, z^{\prime} A, \cdots, z^{\prime} A^{n-1}\right)$ is an $E$-generator $z^{\prime \prime}$, and one easily checks that $z^{\prime \prime}=z$. Thus this construction provides a natural one-to-one correspondence of $E$ generators and $E^{\prime}$-generators. Unfortunately the correspondence is not linear and cannot be extended to a correlation; however, it does provide a natural way for applying Theorem 4 , in the cyclic case, to find a nondegenerate form merely by specifying an $E$-generator rather than both an $E$-generator and an $E^{\prime}$-generator. Similar remarks apply in the general case.

If $z$ generates $E$ and $v=v^{*} z$ and $w=w^{*} z$, then the form $g$ given by setting $g(v, w)=$ trace $v^{*} w^{*}$ is clearly an element of $\delta(A)$, and one might expect that it could itself be used to generate $S(A)$ in place of the construction of Theorem 4. However, $g$ is degenerate in some cases, as shown below, and in any event it is no more "natural" than Theorem 4 , since one must still prescribe $z$ in order to compute $v^{*}$ and $w^{*}$.

Let $\left(u_{1}, \cdots, u_{n}\right)$ be a left canonical basis of $E,\left(u_{1}^{\prime}, \cdots, u_{n}^{\prime}\right)$ its dual basis, and define $v^{*}$ and $w^{*}$ by setting $v=v^{*} u_{1}$ and $w=w^{*} u_{1}$. Then any of 
the $n^{2}$ forms $f_{i j}$ given by setting $f_{i j}(v, w)=\left\langle u_{i}^{\prime}, v^{*} w^{*} u_{j}\right\rangle$ is an element of $s(A)$. One easily shows that

$$
\left(\begin{array}{l}
f_{1 j} \\
\vdots \\
f_{n j}
\end{array}\right)=(A)_{l}^{j-1}\left(\begin{array}{l}
f_{1} \\
\vdots \\
f_{n}
\end{array}\right)
$$

where the forms $f_{1}, \cdots, f_{n}$ span $\delta(A)$ as in Theorem 9 , so that $g=\sum_{j=1}^{n} f_{j j}$ $=\sum_{j=1}^{n}\left(\right.$ trace $\left.A^{j-1}\right) f_{j}$. Hence $g$ is nondegenerate if and only if

$$
\operatorname{det} \sum_{j=1}^{n}\left(\operatorname{trace} A^{j-1}\right) f_{j} \neq 0,
$$

which is an algebraic inequality in $\alpha_{0}, \cdots, \alpha_{n-1}$. For example, if $n=2$ then $g$ is degenerate whenever $4 \alpha_{0}+\alpha_{1}^{2}=0$.

UNIVERSITY OF ILLINOIS,

URBANA, ILL. 